\title{
The pseudokinase SgK223 promotes invasion of pancreatic ductal epithelial cells through JAK1/Stat3 signaling
}

Carole M. Tactacan ${ }^{1,2}$, Yu Wei Phua ${ }^{2}$, Ling Liu², Luxi Zhang ${ }^{1,2}$, Emily S. Humphrey ${ }^{1}$, Mark Cowley ${ }^{1}$, Mark Pinese ${ }^{1}$, Andrew V. Biankin ${ }^{1,3}$ and Roger J. Daly ${ }^{1,2,4^{*}}$

\begin{abstract}
Background: Characterization of molecular mechanisms underpinning development of pancreatic ductal adenocarcinoma (PDAC) may lead to the identification of novel therapeutic targets and biomarkers. SgK223, also known as Pragmin, is a pseudokinase and scaffolding protein closely related to SgK269/PEAK1. Both proteins are implicated in oncogenic tyrosine kinase signaling, but their mechanisms and function remain poorly characterized.
\end{abstract}

Methods: Expression of SgK223 in PDAC and PDAC cell lines was characterized using gene expression microarrays, mass spectrometry (MS)-based phosphoproteomics and Western blotting. SgK223 was overexpressed in human pancreatic ductal epithelial (HPDE) cells via retroviral transduction, and knocked down in PDAC cells using siRNA. Cell proliferation was determined using a colorimetric cell viability assay, and cell migration and invasion using transwells. Expression of markers of epithelial-mesenchyme transition (EMT) was assayed by quantitative PCR. SgK223 and Stat3 signaling was interrogated by immunoprecipitation, Western blot and gene reporter assays. The functional role of specific kinases and Stat3 was determined using selective small molecule inhibitors.

Results: Elevated site-selective tyrosine phosphorylation of SgK223 was identified in subsets of PDAC cell lines, and increased expression of SgK223 detected in several PDAC cell lines compared to human pancreatic ductal epithelial (HPDE) cells and in PDACs compared to normal pancreas. Expression of SgK223 in HPDE cells at levels comparable to those in PDAC did not alter cell proliferation but led to a more elongated morphology, enhanced migration and invasion and induced gene expression changes characteristic of a partial EMT. While SgK223 overexpression did not affect activation of Erk or Akt, it led to increased Stat3 Tyr705 phosphorylation and Stat3 transcriptional activity, and SgK223 and Stat3 associated in vivo. SgK223-overexpressing cells exhibited increased JAK1 activation, and use of selective inhibitors determined that the increased Stat 3 signaling driven by SgK223 was JAK-dependent. Pharmacological inhibition of Stat3 revealed that Stat3 activation was required for the enhanced motility and invasion of SgK223-overexpressing cells.

Conclusions: Increased expression of SgK223 occurs in PDAC, and overexpression of SgK223 in pancreatic ductal epithelial cells promotes acquisition of a migratory and invasive phenotype through enhanced JAK1/Stat3 signaling. This represents the first association of SgK223 with a particular human cancer, and links SgK223 with a major signaling pathway strongly implicated in PDAC progression.

Keywords: Pragmin, SgK269, PEAK1, Tyrosine kinase, Pancreatic cancer

\footnotetext{
*Correspondence: roger.daly@monash.edu

${ }^{1}$ Cancer Research Division, The Kinghorn Cancer Centre, Garvan Institute of

Medical Research, 384 Victoria St, Darlinghurst, Sydney, NSW 2010, Australia

${ }^{2}$ Department of Biochemistry and Molecular Biology, Monash University,

Clayton, VIC 3800, Australia

Full list of author information is available at the end of the article
}

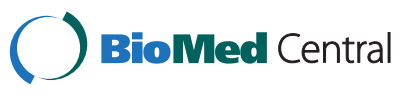

(c) 2015 Tactacan et al. This is an Open Access article distributed under the terms of the Creative Commons Attribution License (http://creativecommons.org/licenses/by/4.0), which permits unrestricted use, distribution, and reproduction in any medium, provided the original work is properly credited. The Creative Commons Public Domain Dedication waiver (http:// creativecommons.org/publicdomain/zero/1.0/) applies to the data made available in this article, unless otherwise stated. 


\section{Background}

Pancreatic ductal adenocarcinoma (PDAC) remains one of most aggressive and lethal of human cancers. The overall 5-year survival rate is $<5 \%$, a statistic that has not changed in almost 50 years [1]. Until recently, gemcitabine was the current standard for post-operative chemotherapy, delaying the development of recurrent disease in some PDAC patients, with a modest improvement in overall survival achieved upon combination with the epidermal growth factor receptor (EGFR) -directed tyrosine kinase inhibitor erlotinib [2]. However, recent studies indicate that the addition of nab-paclitaxel to gemcitabine can provide a significant survival benefit [2], and the combined chemotherapeutic modality Folfirinox has emerged as a more effective treatment than gemcitabine, although at the cost of significant toxicity [2]. Further characterization of the molecular pathways regulating PDAC development and progression may lead to the identification of improved therapeutic strategies, as well as biomarkers that help stratify patients for optimal treatment.

Multiple lines of evidence support a role for deregulated tyrosine kinase signaling in PDAC development and progression. For example, activating KRas mutations represent the earliest known genetic alteration in PDAC [3], and evidence from genetically-modified mouse models support their functional role in this malignancy [4]. Signaling by the EGFR is required for acinar-ductal metaplasia (ADM), an early step in PDAC progression, as well as survival of cells in established ADM and pancreatic intraepithelial neoplasia lesions [5], and overexpression of the EGFR [6] and the related receptor ERBB3 [7] has been detected in PDAC, with aberrant expression being associated with poor prognosis. Alterations can also occur in downstream signaling components. For example, AKT2 gene amplification [8], and loss of the tumour suppressor PTEN [9], occur in this disease. Furthermore, tyrosine phosphorylation of the transcriptional regulator and downstream target of JAK signaling, Stat3, is enhanced in PDAC compared to normal tissue [10], and is associated with poor outcome in resected disease [11]. Importantly, conditional ablation of Stat3 in KRas-driven mouse models of PDAC have confirmed the importance of Stat3 signaling in ADM [12], pancreatic intraepithelial neoplasia (panIN) formation $[12,13]$ and panIN progression and PDAC development [12, 14].

SgK223/Pragmin [15], and SgK269/PEAK1 [16] are large, closely-related proteins that possess a kinase-like domain at their C-termini. However, these kinase-like domains contain substitutions in key motifs characteristic of bona fide kinases, such as the DFG motif responsible for $\mathrm{Mg}^{2+}$-ATP binding, where the aspartate residue is substituted by asparagine. Since both proteins lack nucleotide binding activity based on a thermal shift assay, they likely represent pseudokinases [17]. N-terminal to the pseudokinase domain, both proteins contain tyrosine phosphorylation sites that recruit specific $\mathrm{SH} 2$ and PTB domain-containing effectors, indicating that SgK223 and SgK269 undertake a scaffolding role during tyrosine kinase signaling. For example, SgK223 binds to Csk, a negative regulator of Src, via SgK223 Y411 [18], while SgK269 binds to Grb2 and Shc1 via Y635 and Y1188 to promote proliferative and morphogenic signals, respectively $[19,20]$. Recent work has determined that SgK269 plays a key role during growth factor receptor signaling, mediating a qualitative switch in EGFR output from proliferative/survival signaling to promotion of cell migration/invasion [20].

Importantly, SgK223 and SgK269 both exhibit emerging oncogenic roles. For example, SgK223 promotes cell invasion in colon carcinoma cells exhibiting high Src activity [21], while overexpression of SgK269 promotes growth and aberrant morphogenesis of MCF-10A mammary epithelial cells, and is required for epithelial-to-mesenchymal transition (EMT) and anchorage-independent growth of basal breast cancer cells [19]. In addition, SgK269 is required for efficient tumour formation and metastasis in an orthotopic pancreatic cancer xenograft model [22]. SgK269 is overexpressed in colon, pancreatic and breast cancers relative to normal tissue $[19,22,16]$, but the expression profile of SgK223 in human malignancies is poorly characterized.

In this study we demonstrate that SgK223 exhibits enhanced phosphorylation and/or expression in PDAC cell lines and tumours relative to normal controls. In addition, we identify a novel pathway linking SgK223, Stat 3 and an invasive phenotype during PDAC development. Overall this work provides important new insights into the signaling and oncogenic function of this pseudokinase scaffold.

\section{Results}

\section{SgK223 is overexpressed in pancreatic cancer}

Mass spectrometry-based phosphoproteomic profiling across a wide PDAC cell line panel detected differential phosphorylation of SgK223 Y159 and Y411, suggesting that SgK223 signaling is perturbed in this malignancy (Fig. 1a, b) (Humphrey et al. manuscript in preparation). Three cell lines (MiaPaca2, Panc10.05 and PL45) exhibited relatively high and low levels of tyrosine phosphorylated Y159 and Y411, respectively, while a larger subgroup of 8 cell lines were characterized by increased levels of phosphorylated Y411. These findings led us to assay total SgK223 expression across this panel, and compare this with non-transformed human pancreatic ductal epithelial (HPDE) cells. Western blotting, using a custom rabbit polyclonal antibody, revealed that SgK223 was overexpressed relative to HPDE cells in all pancreatic cancer cell 


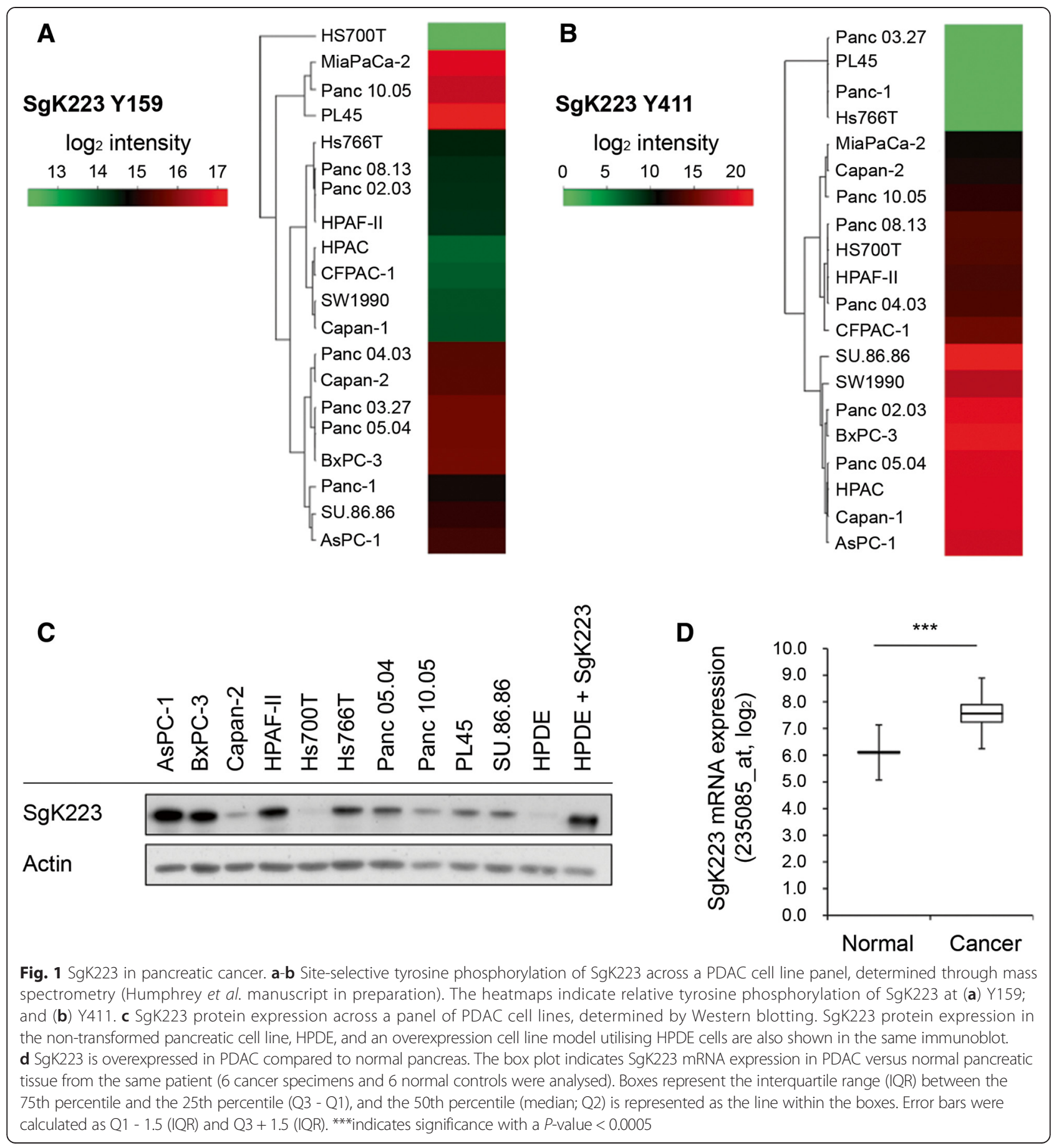

lines tested except Hs700T (Fig. 1c). Of particular note was the overexpression of SgK223 in the cell lines AsPC-1 and $\mathrm{BxPC}-3$, members of the cell line subgroup characterized by high levels of Y411 (Fig. 1c). In order to determine whether SgK223 is overexpressed in primary PDAC, we analysed our own gene expression data for PDAC specimens and macro- and microscopically normal-appearing pancreatic tissue from the same patients [23]. This revealed a marked increase in SgK223 expression in PDAC versus normal controls (Fig. 1d). Thus data from cell line models and human tissue specimens are suggestive of a role for SgK223 in PDAC development and/or progression.

\section{Overexpressing SgK223 in HPDE cells increases cell} migration and invasion

In order to characterize the functional role of SgK223 in pancreatic cancer, we overexpressed this protein in 
HPDE cells via retroviral infection, using a construct encoding rat SgK223 [15]. This led to an expression level comparable to that of AsPC-1 and BxPC-3 pancreatic cancer cells (Fig. 1c). Phosphoproteomic profiling was undertaken to characterize site-selective phosphorylation of the ectopically-expressed SgK223, using BxPC-3 PDAC cells as a positive control. Tyrosine phosphorylation of endogenous SgK223 at Y411 was detected in HPDE and HPDE/SgK223 cells at a comparable level, and at approximately 4-fold higher levels in BxPC-3 cells. In addition, compared with the phosphorylation of endogenous Y411 in HPDEs, a slightly higher level of tyrosine phosphorylation of Y391, the rat equivalent of Y411, was detected in the HPDE/SgK223 cells.
Phosphorylation of rat Y146, equivalent to human Y159, was not detected (data not shown). Therefore, in the context of SgK223 phosphorylation, HPDE/SgK223 cells most closely model the subset of PDAC lines with high phosphorylation of Y411 and low/undetectable phosphorylation of Y159 (eg Capan-1, SW1990, Fig. 1a, b).

SgK223 overexpression altered the morphology of HPDE cells, resulting in an elongated and more refractile mesenchymal-like phenotype (Fig. 2a-c), but did not affect anchorage-dependent cellular proliferation (Fig. 2d). To determine whether SgK223 contributed to a motile and invasive phenotype, transwell assays were undertaken. SgK223 overexpression significantly increased cellular migration (Fig. 2e), and to a greater
A

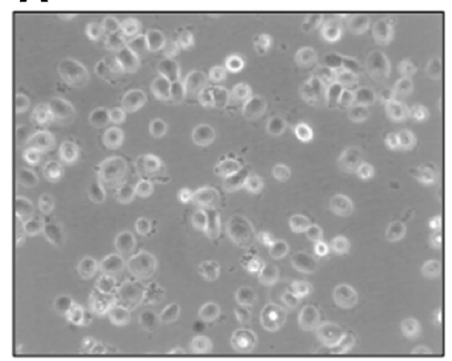

D

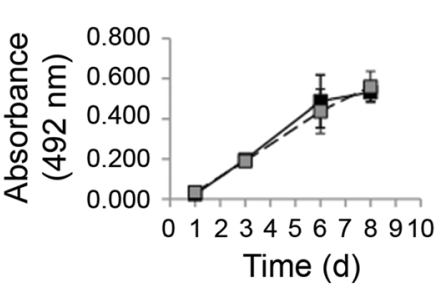

B

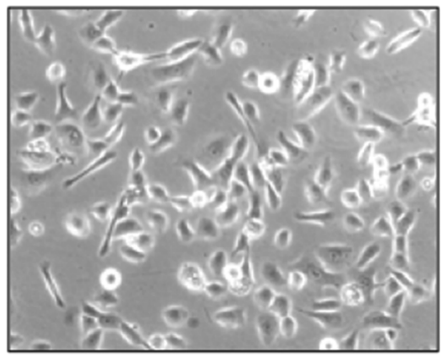

C

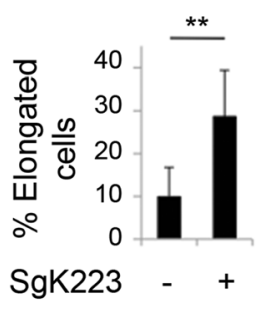

G

$\mathbf{E}$

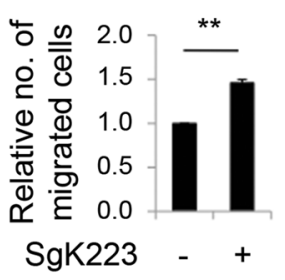

$\mathbf{F}$

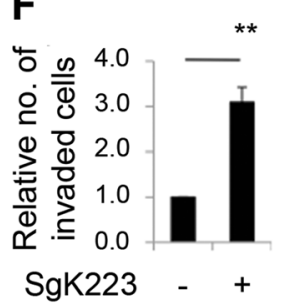

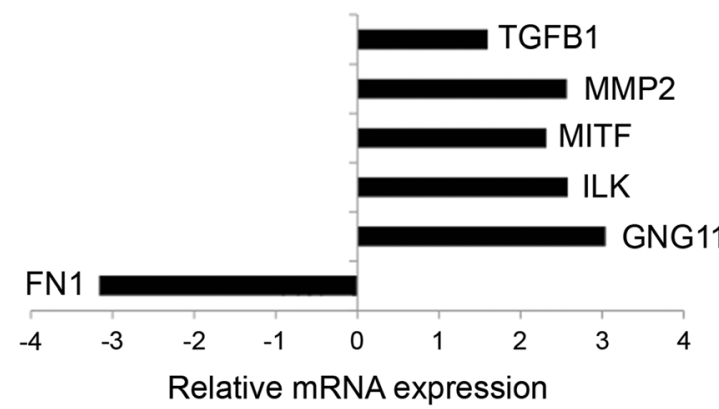

Fig. 2 SgK223 overexpression in HPDE cells enhances cell migration and invasion. SgK223 alters the morphology of HPDE cells grown in monolayer. Light microscope images of HPDE cells containing either: (a) the vector-control; or (b) the SgK223-expression construct. c The relative percentage of elongated cells (cells with lengths double their width) in the vector-control and the SgK223-overexpression cell lines. Data represent mean and the standard error of mean (SEM). **indicates significance with a P-value < 0.005. d SgK223 does not affect the proliferation rates of HPDE cells grown in monolayer. The MTS assay was used to quantify the number of cells, over time. Graph shows the relative proliferation rates between the vector-control (black) and SgK223-overexpressing (grey) HPDE, cell lines. Data points represent mean and SEM at different time points. (E) Relative cell migration. Data are expressed relative to the vector control, which is arbitrarily set at 1.0. Bars represent SEM of three independent experiments, **indicates significance with a $P$-value $<0.005$. $\mathbf{f}$ Relative cell invasion, data are represented as in (e). $\mathbf{g}$ Expression analysis on EMT-associated genes. The histogram indicates relative fold-difference in transcript levels of 6 genes with significant changes in expression in the SgK223-overexpressing, relative to the vector-control, cell line 
extent, enhanced cellular invasion (Fig. 2f). Since acquisition of a mesenchymal morphology and increased motility and invasiveness are suggestive of epithelial-tomesenchymal transition (EMT), we characterized the expression of 84 EMT-related genes using a BD Bioscience $\mathrm{RT}^{2}$ Profiler $^{\mathrm{m}}$ PCR Array. Of the 21 genes on the array that are normally downregulated during EMT, none showed significant change. Of the 63 genes on the array that are normally upregulated during EMT, 6 showed a significant change, and the majority of these $(5 / 6)$ increased in expression (Fig. 2g). The latter included TGFB1 and MMP2. These findings indicate that SgK223 overexpression induces a partial EMT.

\section{The impact of SgK223 on downstream signaling pathways}

Next, we sought to determine how $\operatorname{SgK} 223$ elicits these biological effects by characterizing its impact on known downstream signaling pathways. While the related SgK269 modulates signaling via the focal adhesion proteins p130Cas and paxillin [16], phosphorylation of p130Cas (on Y249) and paxillin (on Y31) was similar in control and SgK223-overexpressing cells (data not shown). In addition, activation of Erk and Akt was similar in control and SgK223-overexpressing cells (Fig. 3a). However, Stat3 tyrosine phosphorylation was enhanced approximately 3 -fold (Fig. 3b). In order to determine whether this was accompanied by an increase in Stat3 transcriptional activity, we undertook reporter assays (Fig. 3c). Indeed, a corresponding increase in expression from a Stat3 reporter construct was observed in SgK223-overexpressing cells. Additionally, knocking down SgK223 in AsPC-1 cells using several independent siRNAs decreased Stat3 tyrosine phosphorylation (Fig. 3d), demonstrating that endogenous high levels of SgK223 in PDAC cells promote Stat3 activation. Since
A

\begin{tabular}{ll} 
SgK223 & -+ \\
\hline SgK223 & \\
Actin & \\
p-ERK112 & \\
(T202N204) \\
ERK1/2 \\
p-AKT \\
(S473) \\
p-AKT \\
(T308) \\
AKT
\end{tabular}

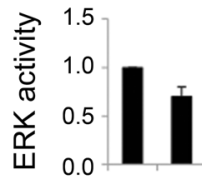

SgK223

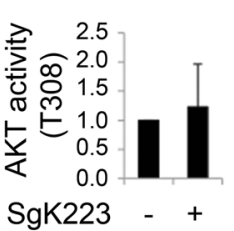

D

\begin{tabular}{|c|c|}
\hline & 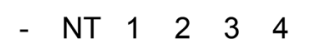 \\
\hline SgK223 & $-\infty-1$ \\
\hline Actin & $-----\cdots$ \\
\hline $\begin{array}{l}\text { p-STAT3 } \\
\text { (Y705) }\end{array}$ & $----1-$ \\
\hline STAT3 & $----\div-$ \\
\hline
\end{tabular}

B

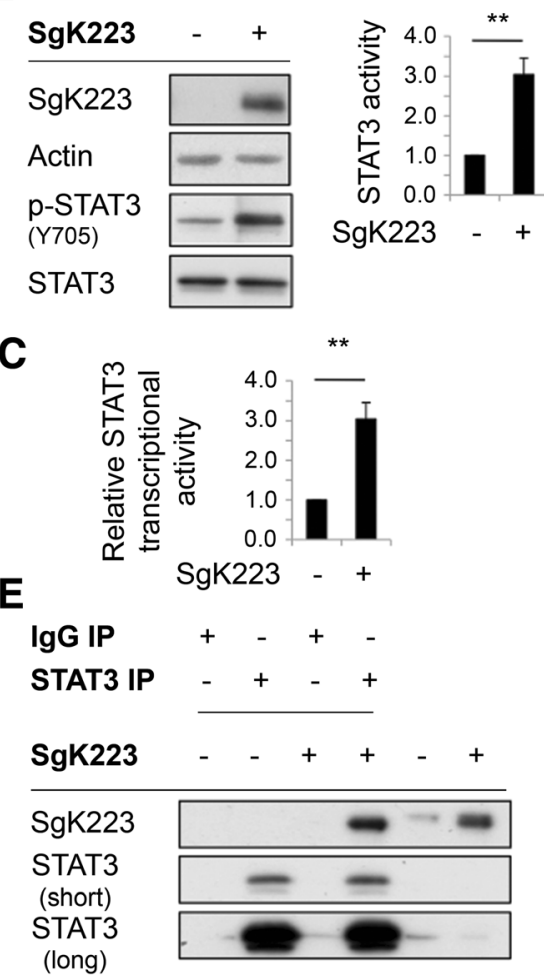

Fig. 3 The effect of SgK223 overexpression on specific signaling pathways. a SgK223 does not affect activation of Erk or Akt. Western blots were undertaken as indicated. Phospho-Erk and -Akt (T308) signals were quantitated by densitometry and normalized for total expression, and expressed relative to the value for control cells, which was arbitrarily set at 1.0. The histograms represent the mean +/- the SE from three independent experiments. b SgK223 enhances Stat3 tyrosine phosphorylation (pY705). Data are normalized and expressed as in the previous panel. **indicates significance with a P-value $<0.005$. c SgK223 enhances Stat3 transcriptional activity. Data are expressed relative to the value for control cells, which was arbitrarily set at 1.0. The histograms represent the mean $+/-$ the SE from three independent experiments. ** indicates significance with a $P$-value < 0.005. d SgK223 knockdown in AsPC-1 PDAC cells decreases Stat3 activation. Two days following transfection using non-targeting (NT) or individual siRNAs directed against SgK223, cell lysates were prepared and Western blotted as indicated. e SgK223 and Stat3 associate in vivo. Control (-) or SgK223-overexpressing (+) HPDE cells were subject to immunoprecipitation (IP) using Stat3 antibodies, or corresponding mouse IgG (negative control). IPs or total cell lysates before IP were Western blotted as indicated. The upper and lower panel of the Stat3 blot represent a short and long exposure, respectively 
SgK223 has a known scaffolding role in signal transduction [18], we determined whether SgK223 and Stat3 associate. Indeed, SgK223 co-immunoprecipitated with Stat3 (Fig. 3e).

\section{SgK223 signals via JAK1 to enhance STAT3 activation}

Next, we set out to determine how SgK223 enhances Stat3 signaling. Known Stat3 kinases include Src family kinases, the EGFR, and the JAKs. While total levels of Src were similar in the SgK223-overexpressing and vector control cells (Fig. 4a), phosphorylation of Src Y416 (Y419 in human Src) on the activation loop was significantly lower in the former cell type, while phosphorylation on the negative regulatory site Y527 (Y530 in human $\mathrm{Src}$ ) was enhanced. Consistent with these observations, expression of Csk, which phosphorylates Src on Y530, was significantly higher upon SgK223 overexpression (Fig. 4a). In addition, treatment of HPDE cells overexpressing SgK223 with the selective Src inhibitor saracatinib did not affect Stat3 phosphorylation (Fig. 4b). These data rule out SFKs as the mediators of enhanced Stat3 phosphorylation in SgK223-overexpressing cells.

With regard to EGFR signaling, SgK223 overexpression increased phosphorylation of the EGFR on Y1068 and decreased Grb2 levels (Fig. 5a). These data were of interest because Y1068 represents a binding site for both Stat3 and Grb2 [24], so these changes may lead to enhanced EGFR signaling through Stat3. Furthermore, SgK223 also increased phosphorylation of the EGFR on Y845, Y1045, Y1148 and Y1173 (Fig. 5b). However, treatment of the SgK223-overexpressing cells with the selective EGFR tyrosine kinase inhibitor erlotinib did not affect Stat3 activation, despite clear inhibition of EGFR
Y1068 phosphorylation (Fig. 5c), indicating that the enhanced Stat3 tyrosine phosphorylation in SgK223overexpressing cells was not mediated by the EGFR.

Next, we characterized the role of the JAKs. Western blotting with appropriate phosphospecific antibodies revealed that JAK1 activity was significantly enhanced upon SgK223 overexpression, while JAK2 and TYK2 activities remained unchanged (Fig. 6a). In addition, treatment of the SgK223-overexpressing cells with the selective JAK inhibitor AG490 led to a dose-dependent reduction of JAK1 and Stat3 phosphorylation, identifying JAK1 as the likely upstream Stat3 kinase in these cells (Fig. 6b). The role of the JAKs in Stat3 phosphorylation was confirmed using the different selective JAK inhibitor CP690550 (Fig. 6c). Of note, although we were able to co-immunoprecipitate Stat3 with SgK223 (Fig. 3e), we could not detect association of JAK1 with SgK223 via this approach.

Since signaling by IL- 6 via the common signal transducing component of the IL6 cytokine receptor family, gp130, represents a key mechanism for activation of JAK/Stat signaling, we assayed the status of this pathway in our model system. A commercial antibody array was used to assay the levels of 36 different cytokines in cell culture supernatants derived from control or SgK223-overexpressing cells. While IL-6 could not be detected in supernatants from either cell pool, expression of several other cytokines, including G-CSF and IL- $1 \alpha$, was detectable but not altered upon SgK223 overexpression (data not shown). In addition, expression of gp130, as determined by Western blotting, was similar in the two cell pools (data not shown). Therefore, enhanced JAK1 activation upon SgK223 overexpression does not reflect increased IL-6 production or gp130 expression.

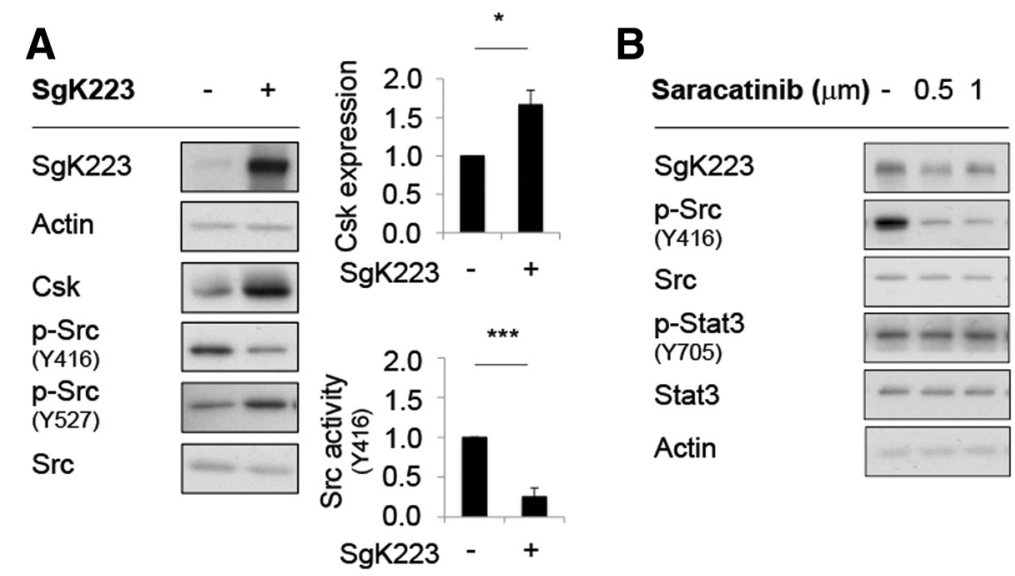

Fig. 4 Enhanced Stat3 activation in SgK223-overexpressing cells is not mediated via Src. a Overexpression of SgK223 in HPDE cells increases Csk expression and decreases Src activity. Data are normalized and expressed as in the previous figures. *indicates significance with a $P$-value $<0.05$, ***indicates significance with a $P$-value $<0.0005$. b The Src inhibitor Saracatinib does not affect Stat3 expression or activation 


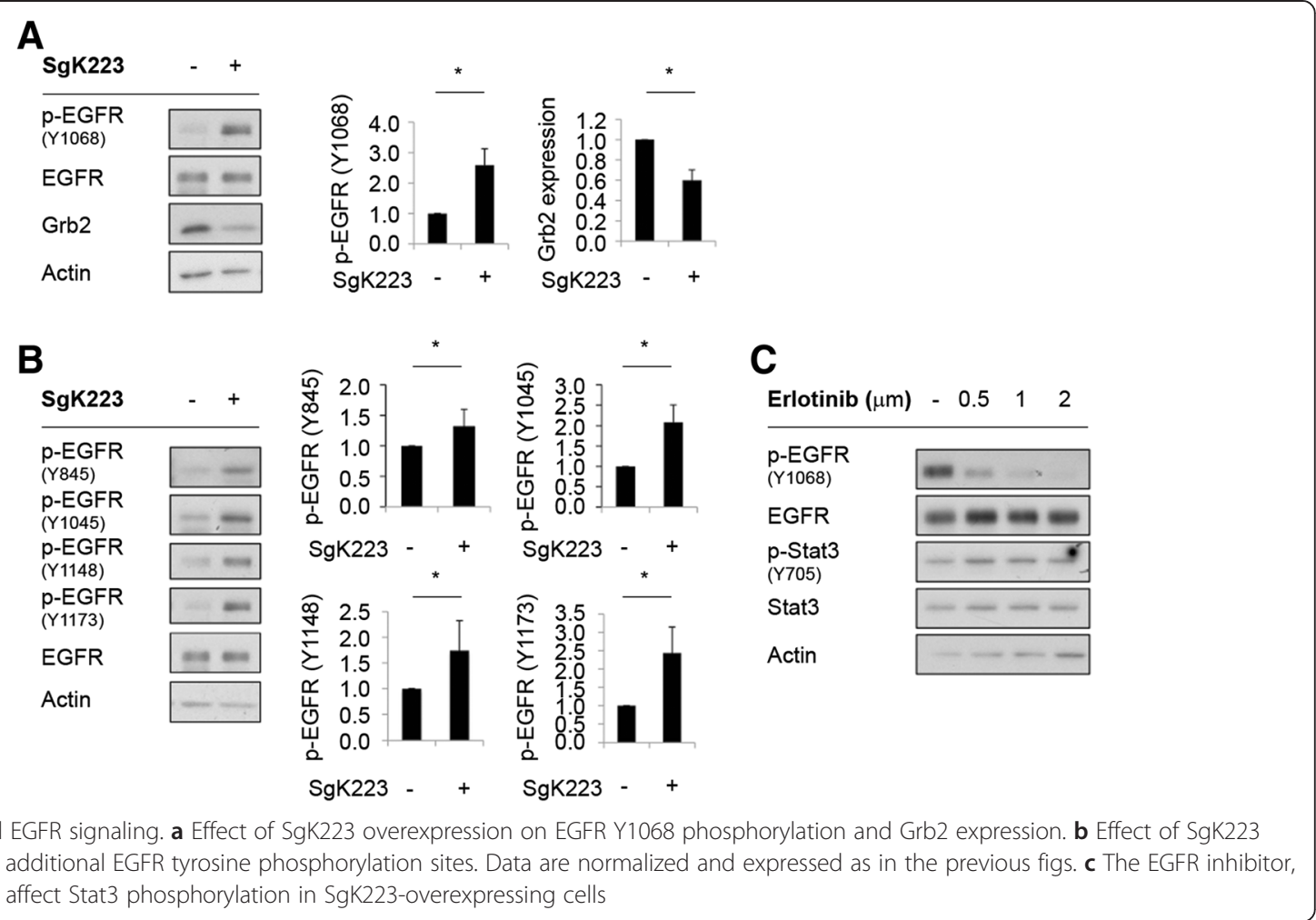

\section{SgK223 enhances cell invasion through STAT3 signaling}

To determine the functional role of Stat3 downstream of SgK223, we utilized the small molecule Stat3 inhibitor STATTIC [25]. Treatment of the SgK223-overexpressing cells with STATTIC markedly reduced Stat3 phosphorylation (Fig. 7a) as well as cell migration (Fig. 7b) and invasion (Fig. 7c). Thus Stat3 activation is required for the enhanced migratory and invasive phenotype of SgK223overexpressing pancreatic ductal epithelial cells.

\section{Discussion}

Several studies have implicated Stat3 activation in PDAC development and progression [12, 11, 13, 14, 10]. Previous reports indicated that the major signaling pathway leading to activation of Stat3 in PDAC is cytokineinduced tyrosine phosphorylation of the signaling coreceptor gp130 and JAK activation [12], and this can occur in a cell autonomous fashion $[12,13]$, as well as via a cell non-autonomous mechanism involving IL-6 production by infiltrating myeloid cells [14]. Increased production and/or exposure to cytokines, and upregulation of gp130, represent identified mechanisms for the enhanced activation of Stat 3 detected in PDAC versus normal tissue [12-14]. Our identification of increased expression of $\mathrm{SgK} 223$ in PDAC versus normal tissue, and demonstration that $\operatorname{SgK} 223$ is sufficient to increase Stat3 activation in HPDE cells and is required for Stat3 phosphorylation in AsPC-1 PDAC cells, now identify aberrant expression of this pseudokinase as an additional mechanism for enhancement of Stat3 signaling in PDAC. While all details of SgK223 action on this pathway have yet to be resolved, our work identifies certain key aspects. First, since JAK1 activation was enhanced in SgK223-overexpressing cells, and selective JAK inhibitors could normalize Stat3 phosphorylation, SgK223 must act upstream of JAK1 to amplify Stat3 signaling. Second, increased production of IL6, and altered expression of gp130, do not appear to be involved. Third, since SgK223 associates with Stat3, this implicates the scaffolding function of SgK223 in regulation of Stat3 phosphorylation, although SgK223 does not itself 'bridge' Stat3 and JAK1. The possibility that SgK223 complexes both gp130 and Stat3, or alters the activity of particular protein tyrosine phosphatases, such as TC-PTP [26], towards Stat3, is currently under investigation. Whatever the mechanism, the phosphorylated Stat3 generated in SgK223-overexpressing cells is competent for nuclear translocation and transcriptional activation of target genes, as confirmed by our reporter assays.

Reflecting their relationship as paralogues, SgK223 and SgK269 exhibit similarities and differences in function and signal output. Both proteins regulate cell morphology, promoting a more elongated phenotype $[19,18]$ and a partial EMT [19]. With regard to the latter process, it is noteworthy that increased expression of either protein led to enhanced expression of MITF, a 

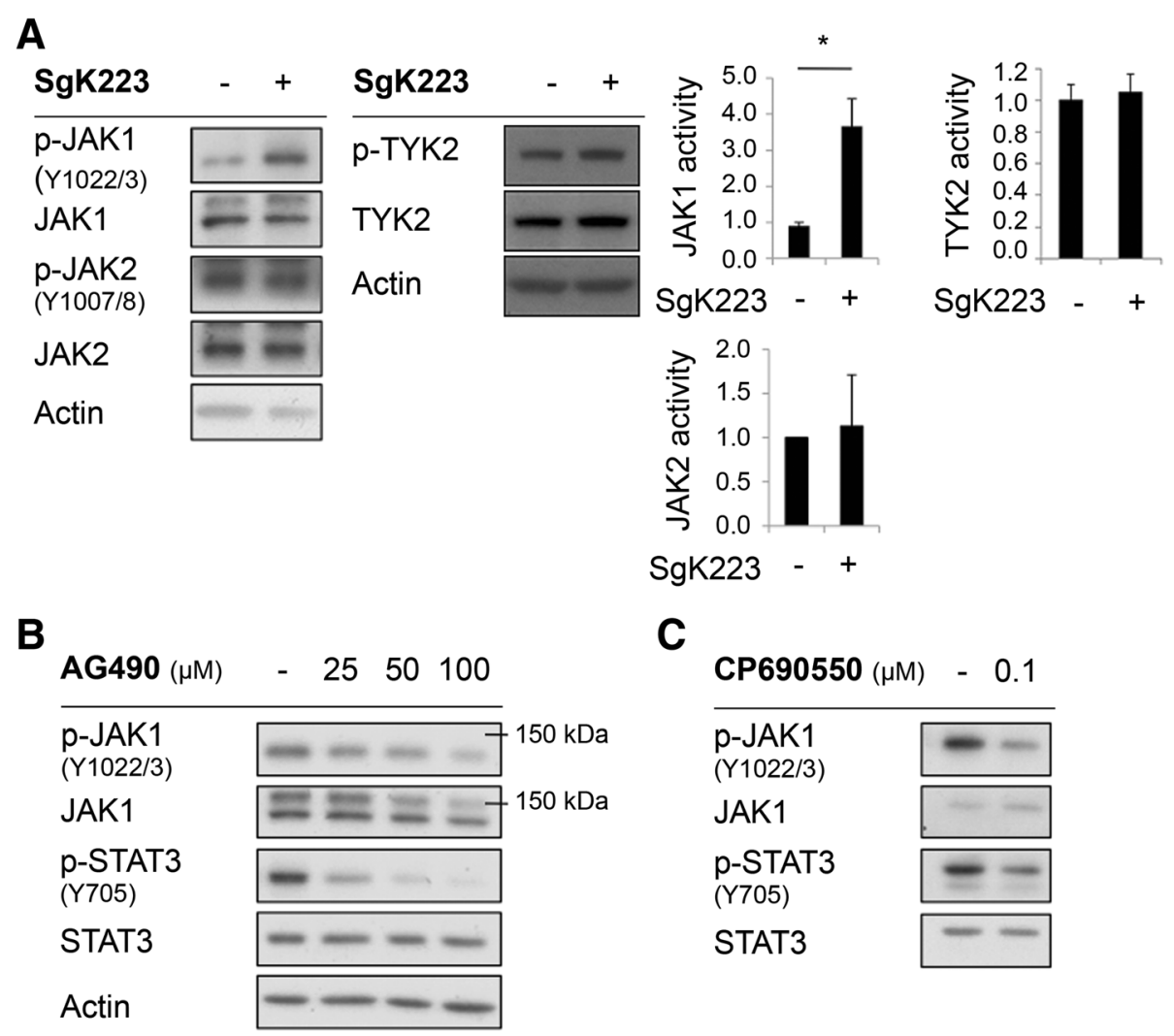

C

CP690550 $(\mu \mathrm{M}) \quad-\quad 0.1$

\begin{tabular}{|c|c|}
\hline $\begin{array}{l}\text { p-JAK1 } \\
\text { (Y1022/3) }\end{array}$ & -1 \\
\hline JAK1 & -7 \\
\hline $\begin{array}{l}\text { p-STAT3 } \\
\text { (Y705) }\end{array}$ & -- \\
\hline STAT3 & -1 \\
\hline
\end{tabular}

Fig. 6 SgK223 signals via JAK1/Stat3. a Overexpression of SgK223 enhances JAK1 activation. Data are normalized and expressed as in Fig. 3. *indicates significance with a P-value < 0.05. b-c Activation of Stat3 in SgK223-overexpressing cells is JAK-dependent. b AG490 or c CP690550 were applied for $2 \mathrm{~h}$ prior to cell lysis. Cell lysates were then Western blotted as indicated

transcription factor that regulates the balance between migration/invasion and proliferation in melanoma cells, and particular TGF $\beta$ family members, that are known to promote acquisition of a mesenchymal phenotype (Fig. 2) $[19,27,28]$. In addition, both SgK223 and SgK269 modulate the tyrosine phosphorylation of specific erbB receptors. The Klemke group [22] demonstrated that SgK269 associated with erbB2, and SgK269 knockdown decreased phosphorylation of this receptor on specific sites, while we observed enhanced EGFR tyrosine phosphorylation upon SgK223 overexpression. However, while SgK269 enhanced site-selective tyrosine phosphorylation of paxillin and p130Cas [16], this direct impact on focal adhesion signaling was not observed in our SgK223 overexpression model. In addition, while SgK269 increases Erk activation [19, 16], this was not
A

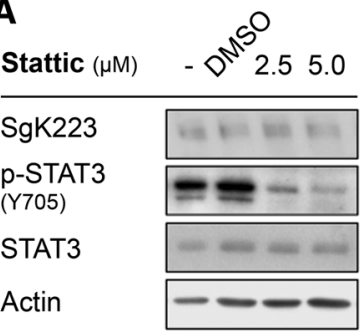

B

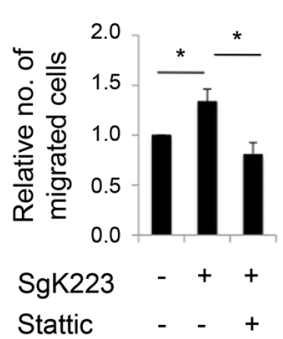

C

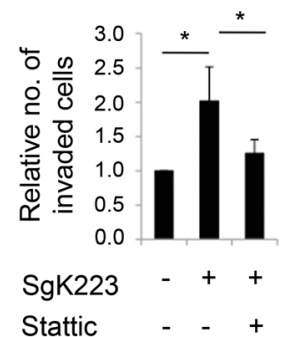

Fig. 7 Promotion of cell migration and invasion by SgK223 is Stat3-dependent. a Inhibition of Stat3 activation using STATTIC. SgK223-overexpressing HPDE cells were treated with Stat3 inhibitor, STATTIC, at the indicated concentrations for $2 \mathrm{~h}$ prior to cell lysis. Cell lysates were then Western blotted as indicated. b-c Effect of STATTIC on cell migration and invasion. Treatment of SgK223-overexpressing cells with STATTIC (2.5 $\mu$ M) reduced migration (b) and invasion (c) to levels comparable to that of untreated control cells. Cell migration and invasion are expressed relative to the value for untreated control HPDE cells, which is arbitrarily set at 1.0. Data represent the mean $+/$ - SEM of three independent experiments. *indicates significance with a $P$-value $<0.05$ 
observed in HPDE cells overexpressing SgK223 (Fig. 3). This likely reflects the absence of a direct Grb2 binding site in SgK223, while SgK269 recruits this adaptor via Y635 [19].

Interestingly, previous studies reported positive regulation of Src by SgK223 and SgK269 [22, 18]. In the case of the former pseudokinase, this reflected sequestration of Csk in the cytosol by SgK223, leading to activation of plasma membrane-localized Src [18]. However, this was demonstrated using transient expression assays in gastric epithelial cells, and in our HPDE cells stably expressing SgK223, Src activity was decreased, likely reflecting a robust increase in Csk expression in the SgK223overexpressing cells, which may represent a negative feedback mechanism. Taking these data and those from the Hatakeyama group in combination, the effect of SgK223 on Src may be context-dependent, and vary according to the relative abundance of Src and its regulators in specific subcellular localizations.

An additional conserved feature of SgK223 and SgK269 is their ability to enhance activation of Stat3 ([19], this paper). In the case of SgK223, we utilized the Stat3 inhibitor Stattic to demonstrate that a key role for SgK223-induced Stat3 signaling is enhanced cellular migration and invasion. The cellular function of Stat3 signaling downstream of SgK269 has yet to be defined, although mutation of Y635 to phenylalanine, which abrogates SgK269-mediated activation of both Erk and Stat3, negatively impacts upon both proliferation in 3D culture and cellular invasion [19]. Consequently, it appears likely that Stat3 represents a common effector utilized by both pseudokinases to drive acquisition of a migratory and invasive phenotype. This highlights an important oncogenic role for both SgK223 and SgK269 in PDAC, given that both are overexpressed in this malignancy (this paper, [22]) and the critical role played by Stat3 in PDAC development [12-14] and metastasis [29]. In addition, the strong links between these pseudokinases and Stat3 signaling indicate that SgK223 and SgK269 represent candidate biomarkers for responsiveness to targeted therapies directed at the JAK/Stat3 pathway.

\section{Conclusions}

Accumulating evidence indicates that pseudokinases play key roles in regulating intracellular signaling pathways by functioning as allosteric regulators or scaffolds [30, 20]. The signaling potential of pseudokinases is also reflected in the growing number of these proteins implicated in human disease, including cancer [31]. In this manuscript, we extend these findings by demonstrating a novel role for the pseudokinase SgK223 in PDAC. Specifically, SgK223 is overexpressed in PDAC relative to normal pancreatic tissue and promotes acquisition of a migratory and invasive phenotype in pancreatic ductal epithelial cells through enhanced JAK1/Stat3 signaling. This represents the first association of SgK223 with a particular human cancer, and links SgK223 with a major signaling pathway strongly implicated in PDAC progression.

\section{Materials and methods \\ Plasmids}

The retroviral expression vector pBabe-puro/myc-Pragmin was a kind gift from Serge Roche, Centre for Biochemical and Macromolecular Research (CRBM), Montpellier Cedex, France [21], and was previously generated in the laboratory led by Manabu Negishi, Kyoto University, Kyoto, Japan [15].

\section{Cell culture and generation of stable cell lines}

HPDE cells stably expressing the murine ecotropic receptor were maintained in Keratinocyte Serum-Free Media (K-SFM), supplemented with $2.5 \mu \mathrm{g}$ human recombinant EGF and $25 \mathrm{mg}$ bovine pituitary extract per litre (Gibco ${ }^{\circ}$, Life Technologies, USA). All pancreatic cancer cell lines were obtained from the American Type Culture Collection (ATCC, USA) and were maintained according to ATCC guidelines. The packaging cell line PlatE was used for retrovirus production, and HPDE cells were infected with retrovirus using the method previously described [32]. Puromycin (Sigma-Aldrich, USA) was used to select for pBabe-puro-positive HPDE cells at a concentration of $200 \mathrm{ng} / \mathrm{ml}$ for 14 days.

\section{Tyrosine phosphorylation profiling}

Global tyrosine phosphorylation profiling was undertaken by immunoaffinity purification and liquid chromatography-tandem mass spectrometry, as previously described [33, 34], using heavy amino acid-labelled synthetic phosphotyrosine-containing peptides for data normalization [35].

\section{Antibodies, inhibitors and siRNA treatment}

All antibodies were from Cell Signaling Technology, except Actin (Santa Cruz, USA) and SgK223. The affinity-purified rabbit antibody selective for SgK223 was generated by Cambridge Research Biochemicals Ltd. by standard procedures. [C]-PTDHSNSTTWHRLHPTD GS-amide was used as the antigen. The following inhibitors were used in this study: Erlotinib hydrochloride (Symansis, USA); AG490, CP690550 (Tocris Bioscience, USA); AZD0530 (saracatinib) (a kind gift from Ian Steet, Cancer Therapeutics CRC, Vic, Australia); and STAT3 Inhibitor V, STATTIC (Calbiochem, Merck Millipore, Germany). Cells were treated with inhibitors for $2 \mathrm{~h}$ at the indicated concentrations prior to cell lysis for immunoblot analysis. The Universal Negative Control \#1 (SIC001) and the SgK223selective siRNAs (SASI_Hs02_0031603-3 to -6) were 
obtained from Sigma-Aldrich. siRNA was applied to cells (final concentration, $5 \mathrm{nM}$ ) using Lipofectamine 2000 (Invitrogen, USA) transfection reagent using the method previously described [32].

\section{Immunoprecipitation and immunoblotting}

Cell lysates for immunoprecipitation and immunoblotting were prepared using normal lysis buffer [36]. Both Myc-Tag (\#2272, Cell Signaling Technology) and SgK223 (Cambridge Research Biochemicals Ltd) antibodies were used to confirm expression from the pBabe-puro/mycPragmin plasmid in HPDE cells.

\section{Cell proliferation, migration and invasion assays}

Cell proliferation assays were conducted using MTS reagent as previously described [32]. For migration and invasion assays, HPDE cells were starved for $24 \mathrm{~h}$ with K-SFM without supplements, prior to starting the assays. Assays were performed using cell culture inserts with $8 \mu \mathrm{M}$ pore size PET filters designed for 24-well plates, uncoated (BD Falcon ${ }^{\text {th }}$ Cell Culture Inserts; BD Biosciences) and coated in Matrigel (BD Biocoat ${ }^{\mathrm{Tm}}$ Growth Factor Reduced MATRIGEL ${ }^{\mathrm{ma}}$ Invasion Chamber; BD Biosciences), for assaying migration and invasion, respectively. For invasion assays, the Matrigel was rehydrated with K-SFM without supplements, for $2 \mathrm{~h}$ at $37{ }^{\circ} \mathrm{C}$ in $5 \% \mathrm{CO}_{2}$, prior to cell seeding. The lower compartment of each well was filled with $0.75 \mathrm{ml}$ of K-SFM supplemented with EGF and bovine pituitary extract. The upper compartment, within the inserts, was filled with $0.5 \mathrm{ml}$ of resuspended cell solution $\left(2.5 \times 10^{4}\right.$ cells $/ 0.5 \mathrm{ml}$ of K-SFM without supplements). Cells were subsequently incubated for $24 \mathrm{~h}$ at $37^{\circ} \mathrm{C}$ in $5 \%$ $\mathrm{CO}_{2}$ to allow migration/invasion through the filter, after which, remaining cells in the upper compartment were removed by swabbing with a cotton tip. The filter from the insert was subsequently stained using Diff Quick solution (Lab Aids Pty. Ltd., Australia). Stained cells that had migrated/invaded through to the underside of the filter were subsequently photographed and quantified using ImageJ software (version 10.2).

\section{Quantitative RT-PCR analysis of EMT genes}

The RNeasy Kit (Qiagen) was used to extract RNA and reverse transcription was conducted with the RT2 First Strand Kit (Qiagen). The RT2 Profiler PCR Array for Human EMT (PAHS-0902E-4; Qiagen) was used for gene expression profiling and quantitative RT-PCR was carried out on an Applied Biosystems ABI 7900 qPCR machine. Data were analyzed using the $2^{\Delta \Delta \mathrm{Ct}}$ method.

\section{STAT3 reporter assay}

The pGL4.23/APRE-luc construct used in this assay was a kind gift from Matthias Ernst (Walter and Eliza Hall Institute of Medical Research, Melbourne, VIC, Australia).
The construct was made by inserting $8 \times$ APRE sites into a firefly luciferase pGL4.23 (Promega, USA) vector, using a method modified from Takeda, T. et al. (1994) [37]. A renilla luciferase pRL-TK (Promega) vector was used as the corresponding co-transfection control. Untransfected cells were used as negative controls, while IL-6-treated cells were used as positive controls. Positive control cells were treated with IL-6 $(100 \mathrm{ng} / \mathrm{ml})$ for $6 \mathrm{~h}$ prior to the measurement of the assay on day three. On day one, HPDE_pBabepuro and HPDE_pBabe-puro/myc-Pragmin cells were seeded, in replicate, at $2 \times 10^{5}$ cells/well, in a 24-well plate. On day two, $2 \mu \mathrm{g}$ of pGL4.23/APRE-luc (firefly) and $0.2 \mu \mathrm{g}$ of pRL-TK (renilla) vectors were co-transfected into the cells using Lipofectamine 2000 (Invitrogen) according to the transfection reagent manufacturer instructions. On day three, cells were lysed, and the luminescence from firefly and renilla luciferase activity was measured using reagents from the Dual-Luciferase ${ }^{\circ}$ Reporter Assay System (Promega) and read on a FLUOstar OPTIMA (BMG Labtech, Germany).

\section{Statistical tests}

These were by standard t-tests, unless otherwise indicated.

\section{Abbreviations}

ADM: Acinar-ductal metaplasia; EGFR: Epidermal growth factor receptor; EMT: Epithelial-to-mesenchymal transition; HPDE: Human pancreatic ductal epithelial; IP: Immuneprecipitation; IQR: Interquartile range; panIN: pancreatic intraepithelial neoplasia; PDAC: Pancreatic ductal adenocarcinoma; SE: Standard error; SEM: Standard error of mean.

\section{Competing interests}

The authors of this manuscript have no conflicts of interest to declare.

\section{Authors' contributions}

CMT characterised SgK223 expression in PDAC cell lines, manipulated SgK223 expression and undertook cellular and signaling assays, and drafted the manuscript. YWP, LL and LZ undertook additional experimentation involving cell signalling assays and phosphoproteomic analysis on the HPDE/ SgK223 cells. ESH performed the phosphoproteomic profiling across the PDAC panel and analysed the resulting data. MC and MP analysed the gene expression data and undertook statistical analyses. RJD conceived of the study, participated in its design and coordination and finalized writing of the manuscript. AVB contributed to project co-ordination. All authors read and approved the final manuscript.

\section{Acknowledgements}

This work was funded by the National Health and Medical Research Council of Australia. CMT and ESH were recipients of Australian Postgraduate Awards and Postgraduate Scholarships from Cancer Institute New South Wales.

\section{Author details}

${ }^{1}$ Cancer Research Division, The Kinghorn Cancer Centre, Garvan Institute of Medical Research, 384 Victoria St, Darlinghurst, Sydney, NSW 2010, Australia. ${ }^{2}$ Department of Biochemistry and Molecular Biology, Monash University, Clayton, VIC 3800, Australia. ${ }^{3}$ Wolfson Wohl Cancer Research Centre, Institute of Cancer Sciences, University of Glasgow, Scotland G61 1BD, UK. ${ }^{4}$ Department of Biochemistry and Molecular Biology, School of Biomedical Sciences, Monash University, Level 1, Building 77, 23 Innovation Walk, Monash, VIC 3800, Australia.

Received: 19 January 2015 Accepted: 15 July 2015

Published online: 29 July 2015 


\section{References}

1. Jemal A, Siegel R, Xu J, Ward E. Cancer statistics, 2010. CA Cancer J Clin. 2010;60(5):277-300. doi:10.3322/caac.20073.

2. Paulson AS, Tran Cao HS, Tempero MA, Lowy AM. Therapeutic advances in pancreatic cancer. Gastroenterology. 2013;144(6):1316-26. doi:10.1053/ j.gastro.2013.01.078.

3. Neuzillet C, Hammel P, Tijeras-Raballand A, Couvelard A, Raymond E. Targeting the Ras-ERK pathway in pancreatic adenocarcinoma. Cancer Metastasis Rev. 2013;32(1-2):147-62. doi:10.1007/s10555-012-9396-2.

4. Mazur PK, Siveke JT. Genetically engineered mouse models of pancreatic cancer: unravelling tumour biology and progressing translational oncology. Gut. 2012;61(10):1488-500. doi:10.1136/gutjnl-2011-300756.

5. Perera RM, Bardeesy N. Ready, set, go: the EGF receptor at the pancreatic cancer starting line. Cancer Cell. 2012;22(3):281-2. doi:10.1016/.j.ccr.2012.08.019.

6. Ueda S, Ogata S, Tsuda H, Kawarabayashi N, Kimura M, Sugiura Y. The correlation between cytoplasmic overexpression of epidermal growth factor receptor and tumor aggressiveness: poor prognosis in patients with pancreatic ductal adenocarcinoma. Pancreas. 2004;29(1):e1-8.

7. Friess H, Yamanaka Y, Kobrin MS, Do DA, Buchler MW, Korc M. Enhanced erbB-3 expression in human pancreatic cancer correlates with tumor progression. Clin Cancer Res. 1995;1(11):1413-20.

8. Ruggeri BA, Huang L, Wood M, Cheng JQ, Testa JR. Amplification and overexpression of the AKT2 oncogene in a subset of human pancreatic ductal adenocarcinomas. Mol Carcinog. 1998;21(2):81-6.

9. Foo WC, Rashid A, Wang H, Katz MH, Lee JE, Pisters PW, et al. Loss of phosphatase and tensin homolog expression is associated with recurrence and poor prognosis in patients with pancreatic ductal adenocarcinoma. Hum Pathol. 2013;44(6):1024-30. doi:10.1016/j.humpath.2012.09.001.

10. Scholz A, Heinze S, Detjen KM, Peters M, Welzel M, Hauff $P$, et al. Activated signal transducer and activator of transcription 3 (STAT3) supports the malignant phenotype of human pancreatic cancer. Gastroenterology. 2003;125(3):891-905.

11. Denley SM, Jamieson NB, McCall P, Oien KA, Morton JP, Carter CR, et al. Activation of the IL-6R/Jak/stat pathway is associated with a poor outcome in resected pancreatic ductal adenocarcinoma. J Gastrointest Surg. 2013;17(5):887-98. doi:10.1007/s11605-013-2168-7.

12. Corcoran RB, Contino G, Deshpande V, Tzatsos A, Conrad C, Benes CH, et al. STAT3 plays a critical role in KRAS-induced pancreatic tumorigenesis. Cancer Res. 2011;71(14):5020-9. doi:10.1158/0008-5472.CAN-11-0908.

13. Fukuda A, Wang SC, Morris JP, Folias AE, Liou A, Kim GE, et al. Stat3 and MMP7 contribute to pancreatic ductal adenocarcinoma initiation and progression. Cancer Cell. 2011;19(4):441-55. doi:10.1016/j.ccr.2011.03.002.

14. Lesina M, Kurkowski MU, Ludes K, Rose-John S, Treiber M, Kloppel G, et al. Stat3/Socs3 activation by IL-6 transsignaling promotes progression of pancreatic intraepithelial neoplasia and development of pancreatic cancer. Cancer Cell. 2011;19(4):456-69. doi:10.1016/j.ccr.2011.03.009.

15. Tanaka $H$, Katoh $H$, Negishi M. Pragmin, a novel effector of Rnd2 GTPase, stimulates RhoA activity. J Biol Chem. 2006;281(15):10355-64. doi:10.1074/ jbc.M511314200.

16. Wang Y, Kelber JA, Tran Cao HS, Cantin GT, Lin R, Wang W, et al. Pseudopodium-enriched atypical kinase 1 regulates the cytoskeleton and cancer progression [corrected]. Proc Natl Acad Sci U S A. 2010:107(24):10920-5. doi:10.1073/pnas.0914776107.

17. Murphy JM, Zhang Q, Young SN, Reese ML, Bailey FP, Eyers PA, et al. A robust methodology to subclassify pseudokinases based on their nucleotide-binding properties. Biochem J. 2014;457(2):323-34. doi:10.1042/ BJ20131174.

18. Safari F, Murata-Kamiya N, Saito Y, Hatakeyama M. Mammalian Pragmin regulates Src family kinases via the Glu-Pro-lle-Tyr-Ala (EPIYA) motif that is exploited by bacterial effectors. Proc Natl Acad Sci U S A. 2011;108(36):14938-43. doi:10.1073/pnas.1107740108

19. Croucher DR, Hochgrafe F, Zhang L, Liu L, Lyons RJ, Rickwood D, et al. Involvement of Lyn and the atypical kinase SgK269/PEAK1 in a basal breast cancer signaling pathway. Cancer Res. 2013;73(6):1969-80. doi:10.1158/00085472.CAN-12-1472.

20. Zheng Y, Zhang C, Croucher DR, Soliman MA, St-Denis N, Pasculescu A, et al. Temporal regulation of EGF signalling networks by the scaffold protein Shc1. Nature. 2013:499(7457):166-71. doi:10.1038/nature12308.

21. Leroy C, Fialin C, Sirvent A, Simon V, Urbach S, Poncet J, et al. Quantitative phosphoproteomics reveals a cluster of tyrosine kinases that mediates SRC invasive activity in advanced colon carcinoma cells. Cancer Res. 2009:69(6):2279-86. doi:10.1158/0008-5472.CAN-08-2354.

22. Kelber JA, Reno T, Kaushal S, Metildi C, Wright T, Stoletov K, et al. KRas induces a Src/PEAK1/ErbB2 kinase amplification loop that drives metastatic growth and therapy resistance in pancreatic cancer. Cancer Res. 2012:72(10):2554-64. doi:10.1158/0008-5472.CAN-11-3552.

23. Segara D, Biankin AV, Kench JG, Langusch CC, Dawson AC, Skalicky DA et al. Expression of HOXB2, a retinoic acid signaling target in pancreatic cancer and pancreatic intraepithelial neoplasia. Clin Cancer Res. 2005;11(9):3587-96. doi:10.1158/1078-0432.CCR-04-1813.

24. Zhang T, Ma J, Cao X. Grb2 regulates Stat3 activation negatively in epidermal growth factor signalling. Biochem J. 2003;376(Pt 2):457-64. doi:10.1042/BJ20030668.

25. Schust J, Sperl B, Hollis A, Mayer TU, Berg T. Stattic: a small-molecule inhibitor of STAT3 activation and dimerization. Chem Biol. 2006;13(11):1235-42. doi:10.1016/ j.chembiol.2006.09.018.

26. Shields BJ, Wiede F, Gurzov EN, Wee K, Hauser C, Zhu HJ, et al. TCPTP regulates SFK and STAT3 signaling and is lost in triple-negative breast cancers. Mol Cell Biol. 2013;33(3):557-70. doi:10.1128/MCB.01016-12.

27. Hsiao JJ, Fisher DE. The roles of microphthalmia-associated transcription factor and pigmentation in melanoma. Arch Biochem Biophys. 2014 doi:10.1016/j.abb.2014.07.019.

28. Zavadil J, Bottinger EP. TGF-beta and epithelial-to-mesenchymal transitions Oncogene. 2005;24(37):5764-74. doi:10.1038/sj.onc.1208927.

29. Wei D, Le X, Zheng L, Wang L, Frey JA, Gao AC, et al. Stat3 activation regulates the expression of vascular endothelial growth factor and human pancreatic cancer angiogenesis and metastasis. Oncogene. 2003;22(3):319-29. doi:10.1038/sj.onc.1206122

30. Zeqiraj E, van Aalten DM. Pseudokinases-remnants of evolution or key allosteric regulators? Curr Opin Struct Biol. 2010;20(6):772-81. doi:10.1016/ j.sbi.2010.10.001.

31. Reiterer V, Eyers PA, Farhan H. Day of the dead: pseudokinases and pseudophosphatases in physiology and disease. Trends Cell Biol. 2014;24(9):489-505. doi:10.1016/j.tcb.2014.03.008

32. Croucher DR, Rickwood D, Tactacan CM, Musgrove EA, Daly RJ. Cortactin modulates RhoA activation and expression of Cip/Kip cyclin-dependent kinase inhibitors to promote cell cycle progression in 11q13-amplified head and neck squamous cell carcinoma cells. Mol Cell Biol. 2010;30(21):5057-70. doi:10.1128/MCB.00249-10.

33. Hochgrafe F, Zhang L, OToole SA, Browne BC, Pinese M, Porta Cubas A, et al. Tyrosine phosphorylation profiling reveals the signaling network characteristics of Basal breast cancer cells. Cancer Res. 2010;70(22):9391-401. doi:10.1158/0008-5472.CAN-10-0911

34. Rikova K, Guo A, Zeng Q, Possemato A, Yu J, Haack H, et al. Global survey of phosphotyrosine signaling identifies oncogenic kinases in lung cancer. Cell. 2007;131(6):1190-203. doi:10.1016/j.cell.2007.11.025.

35. Ali NA, Wu J, Hochgrafe F, Chan H, Nair R, Ye S, et al. Profiling the tyrosine phosphoproteome of different mouse mammary tumour models reveals distinct, model-specific signaling networks and conserved oncogenic pathways. Breast Cancer Res. 2014;16(5):437. doi:10.1186/s13058-014-0437-3.

36. Brummer T, Schramek D, Hayes VM, Bennett HL, Caldon CE, Musgrove EA, et al. Increased proliferation and altered growth factor dependence of human mammary epithelial cells overexpressing the Gab2 docking protein. J Biol Chem. 2006;281(1):626-37. doi:10.1074/jbc.M509567200.

37. Takeda T, Nakajima K, Kojima H, Hirano T. E1A repression of IL-6-induced gene activation by blocking the assembly of IL- 6 response element binding complexes. J Immunol. 1994;153(10):4573-82. 